\title{
地铁转向架构架台架试验载荷谱编制方法*
}

\author{
张子璠 $^{1}$ 杨广雪 $^{1}$ 李 $^{1}$ 强 $^{1}$ 刘汉文 $^{2}$ \\ (1. 北京交通大学机械与电子控制工程学院 北京 100044 ; \\ 2. 东风汽车公司技术中心 武汉 430058)
}

\begin{abstract}
摘要: 采用国际通用的台架试验规范对地铁转向架构架进行疲劳评估时, 会出现无法完全覆盖线路实际工况的情况。为更准 确地评价构架的疲劳可靠性, 在综合分析构架寿命评估要求和试验台控制方案的基础上, 给出载荷相位关系及加载频率的确 定方法, 根据频域疲劳理论和损伤一致性原则, 提出基于构架载荷和应力的线路实测数据的适用于不同相位多通道加载的加 速程序载荷谱编制方法。损伤计算结果表明, 基于实测数据的载荷谱计算损伤速率已覆盖实测损伤速率, 校准后的损伤比值 范围在 1.04 6.47。将频域法损伤结果与基于时域内准静态叠加法得到的结果进行对比, 结果表明, 频域法和时域法的误差 带在 $8.33 \% \sim 18.60 \%$, 表明采用提出的试验台多通道加载方案, 可以准确反映载荷耦合作用下结构的应力响应。该研究成果 为基于实测数据的地铁转向架构架台架谱的建立提供了新思路。
\end{abstract}

关键词: 转向架构架; 台架试验; 频域疲劳; UIC 标准; 相关性

中图分类号: U270; U270

\section{Compilation Method of Load Spectrum for the Bench Test of Metro Bogie Frame}

\author{
ZHANG Zifan $^{1} \quad$ YANG Guangxue $^{1} \quad$ LI Qiang $^{1} \quad$ LIU Hanwen $^{2}$ \\ (1. School of Mechanical, Electronic and Control Engineering, Beijing Jiaotong University, Beijing 100044; \\ 2. DongFeng Motor Corporation Technical Center, Wuhan 430058)
}

\begin{abstract}
When the international standard of bench test is adopted to evaluate the fatigue of metro bogie frame, the actual working condition of the line will not be completely covered. In order to evaluate the fatigue reliability of the frame more accurately, based on the comprehensive analysis of the requirements of the frame life assessment and the control scheme of the test-bed, the determination method of the load phase relationship and the load frequency is given. According to the fatigue theory in the frequency domain and the principle of damage consistency, the method of acceleration programming load spectrum compilation suitable for different phase and multi channels loading based on the measured data of the line load and stress of the frame is proposed. The damage calculation results show that the damage rate calculated by the load spectrum based on the measured data has covered the measured damage rate, and the calibrated damage ratio ranges from 1.04 to 6.47 . The error band of frequency-domain method and time-domain method is between $8.33 \%$ and $18.60 \%$, which shows that the proposed compilation method of load spectrum for the bench test can accurately reflect the stress response of the structure under load coupling. The research results provide a new idea for the establishment of multi channels bench test spectrum of metro bogie frame based on the measured data.
\end{abstract}

Key words: bogie frame; bench test; frequency domain fatigue; UIC standard; correlation

\section{0 前言}

目前我国的地铁转向架台架试验标准主要参考

* 国家重点基础研究发展计划资助项目 (973 计划, 2016YFB1200404-08)。20190924 收到初稿, 20200227 收到修改稿
UIC615-4，JIS4208 以及 EN13749 等, 然而由于各 国标准均参考本国线路工况，在多年的经验基础上 编制，不完全适用于我国国情，因此编制一套准确 反映我国地铁转向架受载情况的载荷谱, 成为了工 程界亟待解决的难题 ${ }^{[1]}$ 。现阶段国内外已有学者针对 台架谱的编制方法开展了大量工作。邹骅 ${ }^{[2]}$ 在进行了 城际动车组线路跟踪测试的基础上，研究基于实测 
载荷(应力)数据以及试验台标定结果的台架谱编制 方法。李凡松等 ${ }^{[3]}$ 研究了基于核密度函数的载荷雨 流矩阵外推方法, 并根据外推结果给出了动车组车 下设备的 8 级程序谱。HAN 等 ${ }^{[4]}$ 研究了城市磁悬浮 列车构架的受载方式, 提出了适用于新型构架的台 架试验方法, 试验结果证明了该方法的有效性。LU 等 ${ }^{[5]}$ 提出了全尺寸构架加速寿命测试方法, 并在此 基础上编制了基于失效机理一致性原理的三阶加速 度载荷谱。

台架试验的关键在于建立载荷与应力之间的关 系。传统的基于雨流计数的台架谱建立方法由于无 法考虑实测载荷间的耦合作用, 只能用于载荷逐一 顺序加载, 造成了应力响应计算结果误差较大。因 此, 需要找到一种可以考虑耦合作用的多通道加载 方法, 使得应力结果更加接近线路实测情况。另外, 由于线路测试得到的是随机载荷, 若直接转化成台 架加载可用的恒幅程序谱, 则会使载荷系间的相位 关系丢失, 载荷对结构的耦合响应发生改变, 导致 台架试验损伤与线路实测损伤存在差异, 因此需要 对随机谱进行修正 ${ }^{[6-7]}$ 。

综上, 本文以北京地铁某型构架为研究对象, 通过试验台标定及线路跟踪测试获得了实测随机载 荷(应力)-时间历程, 提出了基于振动疲劳理论的多 通道台架谱建立方法。该方法分析了相关性与载荷 相位之间的关系, 对线路实测相关系数进行修正以 实现试验台物理可控; 推导了频域内恒幅载荷以及 等效应力的计算方法, 并根据损伤一致性原则进行 了载荷校准, 得到了适用于多通道加载的程序谱。 结果表明, 基于实测数据的台架谱建立方法可以覆 盖线路实测损伤, 并且可以复现多通道加载条件下 的结构应力响应, 具有一定的工程可行性。

\section{1 频域内应力响应计算方法}

使用载荷谱评定构架疲劳寿命, 关键是建立起 载荷和结构应力间的关系。按照线弹性理论, 在构 架所受载荷为低频准静态前提下, 结构应力与外载 之间的关系可由下式表示 ${ }^{[8-9]}$

$$
\sigma=\sum_{i=1}^{n} k_{i} f_{i}
$$

式中, $k_{i}$ 为第 $i$ 个载荷 $f_{i}$ 与测点之间的 “载荷-应力” 传递系数; $n$ 为载荷数量。对式(1)两边同时取自相 关函数, 得到

$$
E\left(\sigma^{2}\right)=\operatorname{Trace}\left\{\left(\boldsymbol{K E}\left(\boldsymbol{F} \boldsymbol{F}^{\mathrm{T}}\right)\right\}\right.
$$

式中, Trace 表示求矩阵的迹; $\boldsymbol{K}$ 为 “载荷-应力”
传递矩阵, 表示为

$$
\boldsymbol{K}=\left(\begin{array}{cccc}
k_{1}^{2} & k_{1} k_{2} & \ldots & k_{1} k_{n} \\
k_{2} k_{1} & k_{2}^{2} & \ldots & k_{2} k_{n} \\
\vdots & \vdots & & \vdots \\
k_{n} k_{1} & k_{n} k_{2} & \ldots & k_{n}^{2}
\end{array}\right)
$$

根据振动疲劳理论, 得到

$$
\int_{0}^{+\infty} W_{\sigma}(\omega) \mathrm{d} \omega=\int_{0}^{+\infty} \operatorname{Trace}\left\{\boldsymbol{K} W_{\boldsymbol{F}}(\omega)\right\} \mathrm{d} \omega
$$

式中, $W_{\sigma}(\omega)$ 为应力的功率谱密度函数; $W_{F}(\omega)$ 为 载荷的功率谱密度函数。

$$
\begin{aligned}
\text { 对式(4)积分进一步得到 } \\
S_{\sigma}^{2}=\operatorname{Trace}\left\{\boldsymbol{K} \boldsymbol{S}_{F}^{2}\right\}
\end{aligned}
$$

$S_{F}^{2}$ 表示为

$$
\boldsymbol{S}_{F}=\left(\begin{array}{cccc}
S_{11}^{2} & S_{12}^{2} & \ldots & S_{1 n}^{2} \\
S_{21}^{2} & S_{22}^{2} & \ldots & S_{2 n}^{2} \\
\vdots & \vdots & & \vdots \\
S_{n 1}^{2} & S_{n 2}^{2} & \ldots & S_{n n}^{2}
\end{array}\right)
$$

式中, $S_{i i}^{2}(i=1,2, \cdots, n)$ 为随机信号的自功率谱密度 的 0 阶矩, 即信号方差; $S_{i j}^{2}(i=1,2, \cdots, n ; j=1,2, \cdots, n)$ 为随机信号的互功率谱密度的 0 阶矩, 即信号协方 差; $S_{\sigma}^{2}$ 为疲劳控制点的频域等效方差 ${ }^{[10-12]}$ 。

互功率谱的 0 阶矩可由下式计算

$$
S_{i j}^{2}=r_{i j} \sqrt{S_{i i}^{2} S_{j j}^{2}}
$$

式中, $r_{i j}$ 为随机信号的相关系数。

由式(6)可知, 载荷系对测点的响应既包括载荷 对测点的单独响应(即矩阵的主对角元素), 此响应与 时域内建立的分立载荷谱产生的响应相同; 也包括了 各载荷系间的耦合作用, 这种作用可以通过载荷间的 互功率谱密度进行量化, 因此在频域内建立载荷谱可 以克服时域分立载荷谱丢失载荷关联性的缺陷。

\section{2 恒幅载荷推导}

本质上恒幅载荷可以视为窄带过程, 而实测信 号通常为宽带过程, 因此可基于谱分解理论推导等 频次程序谱块 ${ }^{[13]}$ 。根据微元思想, 对于宽带随机过 程, 可认为其由多个频率的窄带过程组成。

高斯窄带过程的雨流幅值服从 Rayleigh 分 布 $^{[14]}$, 损伤期望速率为

$$
\dot{D}_{\mathrm{N}}=v_{0} C^{-1}(\sqrt{2} S)^{m} \Gamma\left(1+\frac{m}{2}\right)
$$

式中, $v_{0}$ 为平均正穿越率; $C$ 和 $m$ 为 $S-N$ 曲线参数; $S$ 为信号标准差; $\Gamma(\cdot)$ 为伽马函数。

对载荷功率谱密度进行区间划分之后, 即可通 
过式(8)得到每个窄带随机过程单独作用时的损伤, 进而通过某种方式将损伤进行累加得到总损伤速 率。通常情况下程序谱载荷的加载频率为一固定值, 这显然与谱分解法各窄带过程中心频率互不重合的 基本思想相矛盾, 所以需要进行频率迁移, 统一各 窄带过程的中心频率为疲劳试验机加载频率, 而迁 移的条件是保证损伤速率不变，即

$$
v_{i} C^{-1}\left(2 S_{i}^{2}\right)^{\frac{m}{2}} \Gamma\left(1+\frac{m}{2}\right)=v_{\mathrm{r}} C^{-1}\left[2\left(S_{\mathrm{r}}^{2}\right)_{i}\right]^{\frac{m}{2}} \Gamma\left(1+\frac{m}{2}\right) \text { (9) }
$$

式中, $S_{i}^{2}$ 为第 $i$ 个窄带过程的方差, $\left(S_{\mathrm{r}}^{2}\right)_{i}$ 为迁移频 率后的第 $i$ 个窄带过程对应的转化方差。

由于高斯窄带过程的中心频率等于 $v_{0}$, 根据 式 (9)可得到第 $i$ 个窄带过程的转化方差为

$$
\left(S_{\mathrm{r}}^{2}\right)_{i}=\left(\frac{f_{\mathrm{r}}}{f_{i}}\right)^{\frac{2}{m}} S_{i}^{2}
$$

式中, $f_{i}$ 为第 $i$ 个窄带过程的中心频率; $f_{\mathrm{r}}$ 为目标频率。

得到了转化方差之后, 便可进行累积各转化方 差得到总的转化方差。由于各窄带过程的带宽没有 重叠, 即窄带过程之间相互独立, 根据统计理论, 相互独立的随机过程和的二阶原点矩也满足线性关 系，即总的转化方差为

$$
S_{\mathrm{r}}^{2}=\sum_{i=1}^{N}\left(S_{\mathrm{r}}^{2}\right)_{i}
$$

式中, $N$ 为窄带过程总个数。

通过以上分析，原宽带随机过程已等效成窄带 过程，损伤期望速率可将参数代入式(8)计算

$$
\dot{D}=f_{\mathrm{r}} C^{-1}\left(\sqrt{2} S_{\mathrm{r}}\right)^{m} \Gamma\left(1+\frac{m}{2}\right)
$$

假设台架试验加载次数为 $N_{0}$, 则试验台损伤为

$$
D=\dot{D} \cdot \frac{N_{0}}{f_{\mathrm{r}}}
$$

对于正弦信号, 其方差可以由下式得出

$$
S_{\mathrm{r}}^{2}=0.5 A^{2}
$$

式中, $A$ 为信号幅值。台架试验施加的载荷幅值为

$$
A=\sqrt{2} S_{\text {r }}
$$

\section{3 程序谱相位计算方法}

由第 1 节可知, 准确得到载荷间的相关系数, 对精确量化载荷间的耦合作用至关重要, 虽然通过 实测载荷-时间历程可得到实测相关系数, 但是实测 载荷编制成台架程序谱之后, 随机相位变成了固定 相位, 即程序载荷无法拥有与线路条件相同的相关
系数; 此外台架谱各载荷间的相关性是相互制约的, 现假设只施加三种载荷, 分别设为 $x, y, z$, 并且假 定 $x$ 与 $y$ 载荷的相关系数为 $1, x$ 与 $z$ 载荷的相关系 数为 1 , 则若要使该台架控制系统物理可实现, 则 $y$ 与 $z$ 间的相关系数也必须为 1 而不能为其他值 ${ }^{[15]}$ ，因 此载荷相关系数需要根据实际情况进行调整。本文 方法为：根据载荷间相关性的大小将载荷系构建成 不同的子系统逐一调整, 子系统构建的依据是系统 间的载荷几乎不相关, 而系统内部的载荷相关性较 高。系统内部载荷相关系数确定的方法为：找到与 所有载荷相关性都较高的载荷, 以它为基准调整相 关系数并确定相位差，进而通过相位差得到各个载 荷间的相关系数。

假设加载波形为正弦波, 设为 $\boldsymbol{x}(t)=A \sin \omega t$, $y(t)=B \sin (\omega t+\phi)$, 式中 $\phi$ 为通道间的初始相位差, $A 、 B$ 为幅值, 方差为 $S_{1}^{2}$ 和 $S_{2}^{2}$ 。根据随机过程相关 理论，两信号间的相位差为

$$
\phi=\arccos \frac{2 R}{A B}
$$

式中, $R$ 为互相关函数。

互相关函数与相关系数的关系可表示为

$$
r=\frac{R}{\sqrt{S_{1}^{2} S_{2}^{2}}}
$$

联立式(15) $(17)$ 得到

$$
\phi=\arccos \frac{2 R}{A B}=\arccos \frac{2 r \sqrt{0.5} A \sqrt{0.5} B}{A B}=\arccos r
$$

因此当两信号的频率相同且不考虑滞后性时， 相位差取决于与信号间的相关系数。

将载荷系划分成不同的子系统之后，还需要考 虑各系统间的相位差。由于属于不同系统的载荷几 乎不相关，从简化控制难度的角度设各系统间的相 关系数为 0 。使载荷间相关系数为 0 可以有两种方 法，一种是载荷同频率，相位差为 $90^{\circ}$; 另一种是 载荷间的频带无重叠。由于一个系统内的载荷本身 存在多个相位差，因此第一种方法较难实现。而试 验台加载可以使各通道保持不同的加载频率，所以 第二种方法是实际可行的。

得到了试验台修正的相关系数之后，即可得到 测点损伤期望速率。由于 4 个子系统相互独立，因 此载荷计算损伤速率采用 $\mathrm{PbP}^{[16]}$ 方法求解, 将多轴 耦合载荷进行解耦得到多个相互独立的等效单轴载 荷，并将等效载荷单独作用的损伤贡献进行非线性 求和来计算多轴过程疲劳损伤。前文已将构架 11 种 载荷解耦成 4 种子系统, 因此可直接非线性累加损 伤，计算公式为 


$$
E(D)=\left(\sum_{i=1}^{4} d_{i}^{\frac{2}{m}}\right)^{\frac{m}{2}}
$$

式中, $d_{i}$ 为通过第 $i$ 个子系统的等效恒幅载荷计算 得到的应力方差, 代入式(8)求得的损伤期望速率。

确定了载荷的方差和相位之后, 根据式(8), 还 需要确定加载频率。频率的确定一方面考虑作动器 实际可承受加载频率, 另一方面, UIC 标准仅简单 规定了当线路中曲线较多时, 单个准静态载荷(如侧 滚)循环包含的动态载荷(如浮沉载荷)循环次数应相 应减少, 但并未具体说明二者的关系, 因此应根据 线路各载荷的实测循环数占总循环数的比例, 按照 此占比确定加载频率, 循环次数多的载荷加载频率 大，循环次数少的载荷加载频率小。

\section{4 载荷损伤一致性校准}

通常来讲, 构架某个部位通过载荷谱计算得到 的损伤速率与该部位实测应力损伤速率存在差异, 因此需要对载荷谱进行校准, 校准的依据是载荷谱 计算损伤速率不低于实测应力损伤速率。为此，根 据有限元计算结果, 预先在构架上了布置用于损伤 校准的测点, 主要集中于构架横纵梁连接部(测点号为 $\mathrm{HZ}$ )、横侧梁连接部(测点号为 $\mathrm{HC}$ )、定位转臂座(测点 号为 $\mathrm{Y}$ )、横向减振器座(测点号为 $\mathrm{HJ}$ )、电机吊座(测 点号为 HD)、及齿轮箱吊座(测点号为 HCL) 等 ${ }^{[17]}$ 。为 方便示意, 下文采用编号 1 11 表示浮沉、侧滚、 扭转、构架横向、构架纵向、制动、菱形、电机垂 向、电机横向、齿轮箱垂向和横向减振器载荷。

“载荷-应力” 传递系数通过试验台标定获得,
将标定后的构架安装于地铁车辆上，采用无人值守 设备进行线路跟踪测试，测试工况包含完整的车辆进 出库以及正线运营等，得到测点实测损伤速率如图 1 所示，载荷系实测方差如图 2 所示，载荷系实测相关 系数如表 1 所示。载荷系中，构架横向载荷的方差最 大, 为 5.08 , 电机横向载荷方差最小, 为 0.02 。

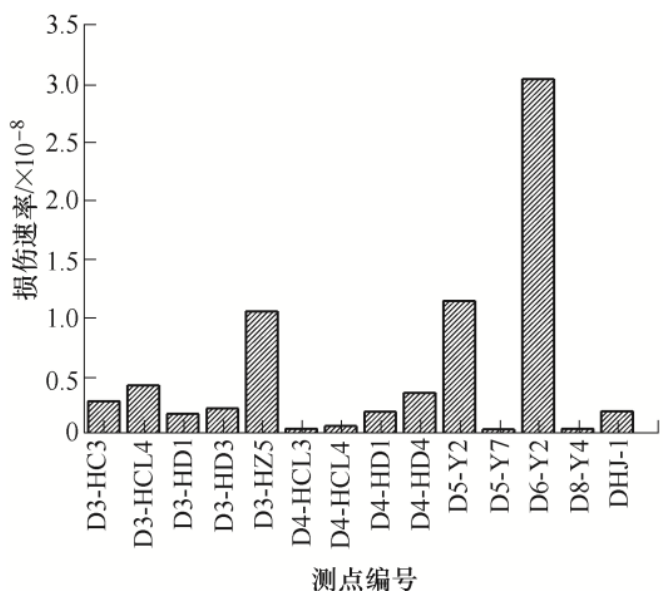

图 1 测点实测损伤速率

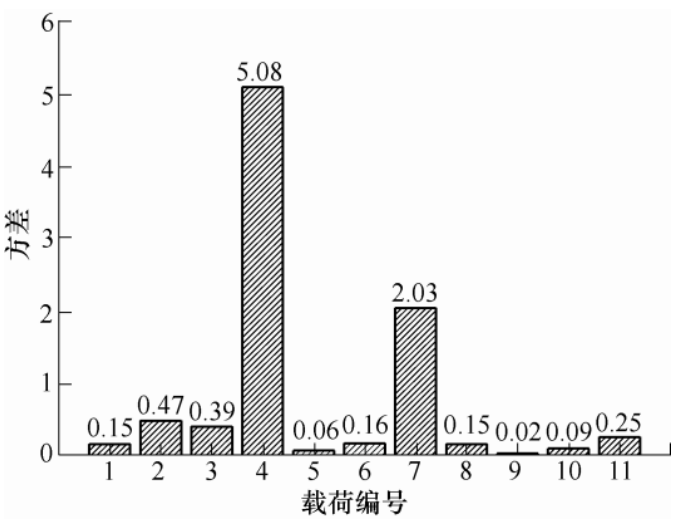

图 2 实测载荷方差

表 1 载荷系线路实测相关系数

\begin{tabular}{cccccccccccc}
\hline 载荷 & 1 & 2 & 3 & 4 & 5 & 6 & 7 & 8 & 9 & 10 & 11 \\
\hline 1 & 1.00 & 0.15 & -0.19 & -0.16 & 0.06 & 0.04 & -0.20 & -0.12 & -0.04 & -0.03 & -0.03 \\
2 & - & 1.00 & 0.06 & 0.55 & -0.03 & 0.14 & -0.69 & -0.04 & 0.10 & 0.05 & 0.60 \\
3 & - & - & 1.00 & 0.60 & 0.05 & -0.15 & 0.72 & 0.10 & -0.15 & -0.06 & -0.53 \\
4 & - & - & - & 1.00 & -0.11 & -0.17 & 0.86 & 0.03 & 0.14 & -0.07 & -0.63 \\
5 & - & - & - & - & 1.00 & -0.35 & 0.12 & -0.05 & -0.06 & -0.37 & -0.07 \\
6 & - & - & - & - & - & 1.00 & -0.18 & -0.03 & 0.08 & 0.39 & 0.09 \\
7 & - & - & - & - & - & - & 1.00 & -0.06 & 0.09 & -0.09 & -0.61 \\
8 & - & - & - & - & - & - & - & 1.00 & 0.38 & -0.02 & -0.02 \\
9 & - & - & - & - & - & - & - & - & 1.00 & -0.04 & 0.11 \\
10 & - & - & - & - & - & - & - & - & - & 1.00 & 0.08 \\
11 & - & - & - & - & - & - & - & - & - & - & 1.00 \\
\hline
\end{tabular}

由表 1 可知, 菱形载荷、横向载荷、扭转载荷、 侧滚载荷和横向减振器载荷之间存在着较高的相关
性，将这五种载荷视作一个子系统，其中菱形载荷 与横向载荷达到 0.86 , 并且另外三种载荷与这两种 
载荷相关性相似, 考虑到台架加载的局限性, 取该 两种载荷的相关系数为 1 , 其他三种载荷取平均值, 调整后结果如表 2 所示, 相位差如表 3 所示。

表 2 第一子系统五种载荷间相关系数

\begin{tabular}{cccccc}
\hline 载荷 & 7 & 4 & 2 & 3 & 11 \\
\hline 7 & 1 & 1 & -0.62 & 0.66 & -0.62 \\
4 & - & 1 & -0.62 & 0.66 & -0.62 \\
2 & - & - & 1 & 0.13 & 1 \\
3 & - & - & - & 1 & 0.13 \\
11 & - & - & - & - & 1 \\
\hline
\end{tabular}

表 3 第一子系统五种载荷间相位差

\begin{tabular}{cccccc}
\hline 载荷 & 7 & 4 & 2 & 3 & 11 \\
\hline 7 & $0^{\circ}$ & $0^{\circ}$ & $128^{\circ}$ & $49^{\circ}$ & $128^{\circ}$ \\
4 & - & $0^{\circ}$ & $128^{\circ}$ & $49^{\circ}$ & $128^{\circ}$ \\
2 & - & - & 0 & $83^{\circ}$ & $0^{\circ}$ \\
3 & - & - & - & 0 & $83^{\circ}$ \\
11 & - & - & - & - & $0^{\circ}$ \\
\hline
\end{tabular}

以此类推, 其他载荷中, 构架纵向载荷、制动 载荷和齿轮箱垂向载荷可视作一个子系统, 电机垂 向载荷和横向载荷为一个子系统, 而浮沉载荷与任 意载荷的相关性都比较低, 所以单独考虑。相关系 数调整结果如表 4、5 所示, 相位差结果如表 $6 、 7$ 所示。

表 4 第二子系统三种载荷间相关系数

\begin{tabular}{cccc}
\hline 载荷 & 5 & 6 & 10 \\
\hline 5 & 1 & -0.36 & -0.36 \\
6 & - & 1 & 1 \\
10 & - & - & 1 \\
\hline
\end{tabular}

\begin{tabular}{ccc}
\hline 表 $\mathbf{5}$ & 第三子系统两种载荷间相关系数 \\
\hline 载荷 & 8 & 9 \\
\hline 8 & 1 & 0.38 \\
9 & - & 1 \\
\hline
\end{tabular}

表 6 第二子系统三种载荷间相位差

\begin{tabular}{cccc}
\hline 载荷 & 5 & 6 & 10 \\
\hline 5 & $0^{\circ}$ & $111^{\circ}$ & $-111^{\circ}$ \\
6 & - & $0^{\circ}$ & $0^{\circ}$ \\
10 & - & - & $0^{\circ}$ \\
\hline
\end{tabular}

表 7 第三子系统两种载荷间相位差

\begin{tabular}{ccc}
\hline 载荷 & 8 & 9 \\
\hline 8 & $0^{\circ}$ & $68^{\circ}$ \\
9 & - & $0^{\circ}$ \\
\hline
\end{tabular}

得到载荷-时间历程之后, 即可通过雨流计数法获 得各载荷的总循环次数, 结果如图 3 所示, 由图 3 可 知, 浮沉载荷循环次数占比最多, 为 $18.3 \%$, 构架
纵向载荷循环次数占比最少, 为 $3.26 \%$, 因此确定 加载频率为: 第一系统 $8 \mathrm{~Hz}$, 第二系统 $6 \mathrm{~Hz}$, 第 三系统 $2 \mathrm{~Hz}$ ，第四系统 $4 \mathrm{~Hz}$ 。

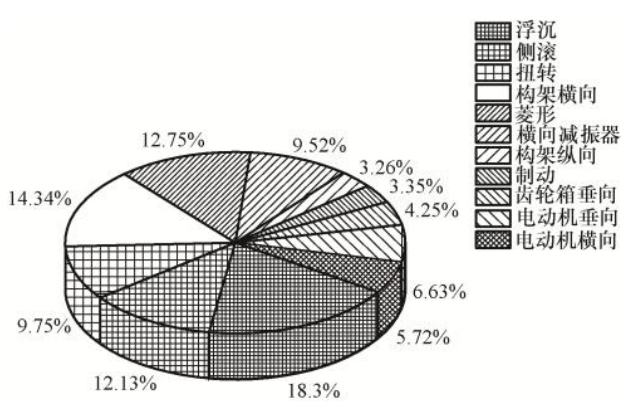

图 3 各载荷雨流频次占比

灰狼算法是 MIRJIALILI 等 ${ }^{[18]}$ 基于自然界中狼 群的群居狩猎方式提出的一种启发式优化算法, 相 较于粒子群等传统启发式算法, 具有受初值影响较 小, 全局收玫性好及稳定性高的优点, 因此本文选 择灰狼算法进行损伤一致校准, 目标函数为载荷谱 计算损伤速率和测点实测应力损伤速率间的均方误 差, 校准后的载荷谱计算损伤速率与实测损伤速率 比如图 4 所示。由图 4 可知, 载荷谱计算损伤速率 已覆盖实测损伤速率, 比值范围在 $1.04 \sim 6.47$, 表 明本文所提出方法建立的多轴加载的台架载荷谱可 以达到线路测试同等条件的考核标准。

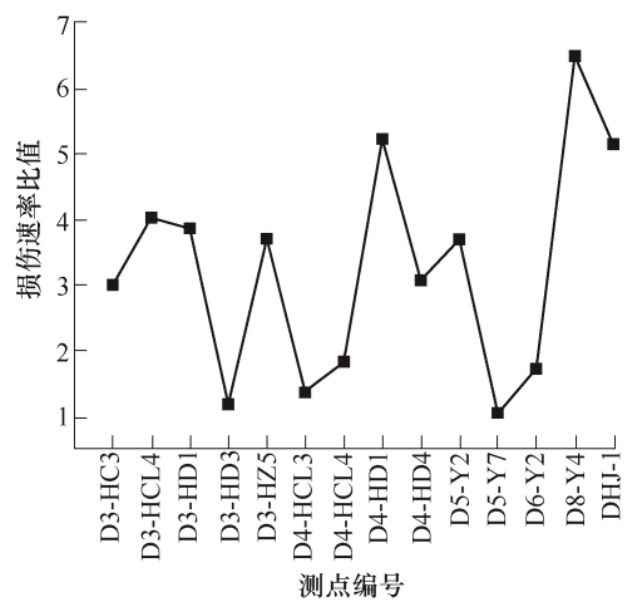

图 4 载荷谱计算损伤速率和实测损伤速率比值

根据台架试验的要求，应确保台架谱计算损伤 可以覆盖构架在全寿命周期内的损伤, 因此需要依 据线路实测损伤外推全寿命周期损伤，并基于后者 推断台架加速损伤速率。地铁目标运营里程为 240 万公里, 根据损伤线性累积法则, 得到实测损伤速 率和台架加速损伤速率的关系为 ${ }^{[19]}$

$$
\frac{\dot{D} \cdot t}{l}=\frac{\dot{D}_{\mathrm{a}} \cdot T}{L}
$$

式中, $\dot{D}$ 为实测损伤速率; $t$ 为线路测试时间, 本 
文选取测试样本为 $2580 \mathrm{~s}$; $l$ 为线路测试里程, 共 计 $23.1 \mathrm{~km} ; \dot{D}_{\mathrm{a}}$ 为台架加速损伤速率; $T$ 为台架试 验时间; $L$ 为目标运营里程。

取台架试验加载次数为焊接结构疲劳极限 200 万次, 以加载频率最低的第三系统为基准, 频率为 $2 \mathrm{~Hz}$, 则台架试验时间为 100 万 $\mathrm{S}$ 。令 $T=1 \times 10^{6} \mathrm{~s}$, 代入式(20), 将 $\dot{D}_{\mathrm{a}}$ 作为未知量, 求解可得测点台架 加速损伤速率, 结果如表 8 所示。

表 8 测点加速损伤期望速率

\begin{tabular}{cc}
\hline 测点 & 加速损伤速率 \\
\hline D3-HC3 & $9.54 \times 10^{-8}$ \\
D3-HCL4 & $7.87 \times 10^{-8}$ \\
D3-HD1 & $8.11 \times 10^{-6}$ \\
D3-HD3 & $1.09 \times 10^{-6}$ \\
D3-HZ5 & $4.83 \times 10^{-7}$ \\
D4-HCL3 & $7.26 \times 10^{-7}$ \\
D4-HCL4 & $2.80 \times 10^{-6}$ \\
D4-HD1 & $4.37 \times 10^{-7}$ \\
D4-HD4 & $3.02 \times 10^{-6}$ \\
D5-Y2 & $1.57 \times 10^{-7}$ \\
D5-Y7 & $5.61 \times 10^{-7}$ \\
D6-Y2 & $9.58 \times 10^{-8}$ \\
D8-Y4 & $4.97 \times 10^{-7}$ \\
DHJ-1 & $9.21 \times 10^{-7}$ \\
\hline
\end{tabular}

加速试验载荷计算方法采用幅值扩大法, 假设 损伤加速前后, 每个子系统对测点造成的损伤占比 保持一致, 可将测点的加速损伤期望速率按照该占 比重新分配给四个子系统, 进而根据式(8)得到加速 后的载荷方差及对应的载荷幅值, 结果如表 9 所示。

表 9 恒幅载荷方差及幅值

\begin{tabular}{ccc}
\hline 载荷 & 方差 & 幅值/kN \\
\hline 1 & 789.63 & 39.74 \\
2 & 437.49 & 29.58 \\
3 & 552 & 33.23 \\
4 & 3897.1 & 88.28 \\
5 & 375.25 & 39.08 \\
6 & 1746.86 & 59.11 \\
7 & 763.44 & 27.4 \\
8 & 1217.74 & 49.35 \\
9 & 271.74 & 23.31 \\
10 & 1414.04 & 53.18 \\
11 & 128.57 & 16.04 \\
\hline
\end{tabular}

文献[2]证明了采用传递系数计算损伤与试验 台动态加载造成的损伤相近, 因此本文采用传递系 数法验证频域内建立的台架谱可以真实反映多通道
加载时测点的应力响应。通过载荷和传递系数按照式 (1)计算应力并在时域内线性叠加, 通过雨流计数法 计算损伤。两种方法得到的各测点损伤结果如图 5 所示。由图 5 可知, 频域法和时域法的结果已十分 接近，误差带在 $8.33 \% \sim 18.60 \%$ ，表明采用本文提 出的试验台多通道加载方案, 可以准确反映载荷耦 合作用下测点的应力响应。

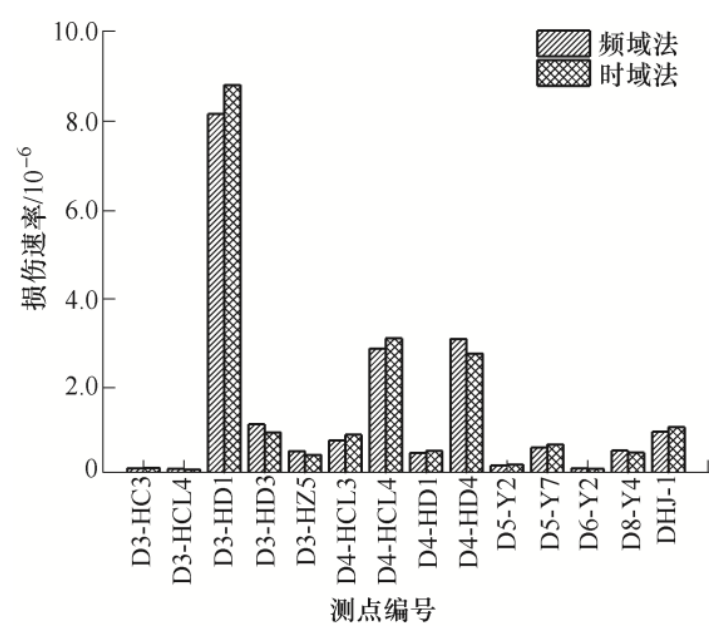

图 5 频域法和时域法损伤对比

\section{5 与标准载荷谱对比分析}

我国轨道机车车辆转向架构架强度试验标准为 TB/T 2368一-2005《动力转向架构架强度试验方法》, 该标准与国际铁路联盟颁布的标准 UIC615-4 基本 等同, 因此本文选用 UIC615-4 标准中规定的载荷 作为参照对比。根据标准得到转向架构架载荷以及 本文所提方法得到的载荷系如表 10 所示。

表 10 本文方法编制的程序谱与标准规定的程序谱

\begin{tabular}{ccc}
\hline 载荷系 & 本文方法载荷幅值 $/ \mathrm{kN}$ & 标准规定载荷幅值 $/ \mathrm{kN}$ \\
\hline 浮沉载荷 & 39.74 & 41.24 \\
侧滚载荷 & 29.58 & 20.61 \\
扭转载荷 & 33.23 & 15.79 \\
构架横向载荷 & 88.28 & 68.96 \\
菱形载荷 & 27.40 & 13.76 \\
减振器载荷 & 16.04 & 5.38 \\
构架纵向载荷 & 39.08 & 36.02 \\
制动载荷 & 59.11 & 40.08 \\
齿轮箱吊座载荷 & 46.73 & 23.84 \\
电机垂向载荷 & 49.35 & 30.21 \\
电机纵向载荷 & 23.31 & 18.17 \\
\hline
\end{tabular}

UIC 标准规定疲劳试验加载分为主要运营载荷 和局部运营载荷两个阶段, 第 1 阶段同时加载浮沉 载荷、横向载荷、侧滚载荷和扭转载荷, 具体加载 
方式及次数如图 6 所示。第 2 阶段为其余各载荷依次 加载。设垂向和横向动载频率为 $8 \mathrm{~Hz}$, 准静态载荷为 $4 \mathrm{~Hz}$, 扭转载荷、纵向载荷和制动载荷为 $2 \mathrm{~Hz}$, 其 余载荷为 $6 \mathrm{~Hz}$, 台架标定载荷-应力传递系数不变, 采用本文方法以及根据标准加载主要运营载荷后的 测点计算损伤如图 7 所示。由图 7 可见, 测点 D3-HC3、 D5-Y2、D5-Y7、D6-Y2、D8-Y4 两种方式计算的损伤 十分接近, 损伤比在 $1.35 \sim 4.56$, 其余测点则相差较大, 笔者分析原因为损伤接近的测点分别位于构架横侧梁 连接部及定位转臂座根部位置, 表明这些测点受主要 运营载荷的影响较大, 而受零部件载荷影响较小, 并 且由于只加载了主要运营载荷, 因此两种方式计算的 损伤接近; 而其余测点分别位于齿轮箱吊座、电机吊 座、制动吊座和减振器吊座, 表明这些位置的损伤主 要是由各零部件振动引起的动载荷贡献, 因此仅加载 主要运营载荷进行疲劳评估在国内的某些线路条件 下会出现欠考核的情况, 此外线路实测中发现的易发 生疲劳失效的部位也大多位于零部件吊座位置, 与本 文分析相吻合。

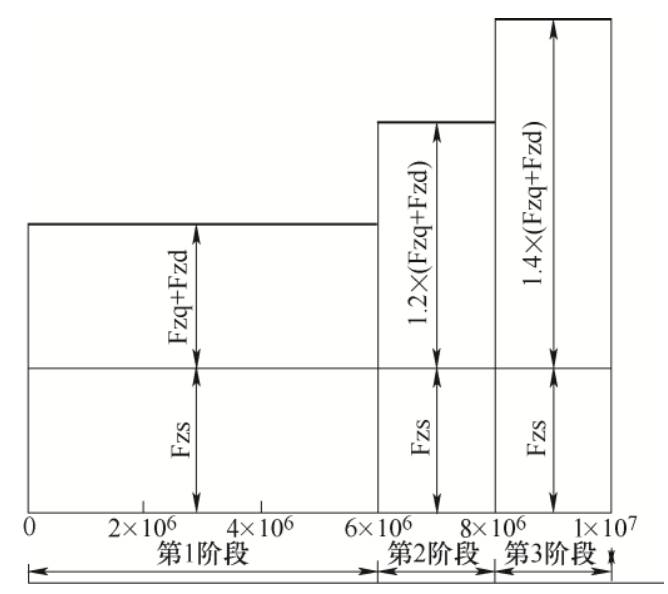

图 6 标准规定的疲劳试验加载方式

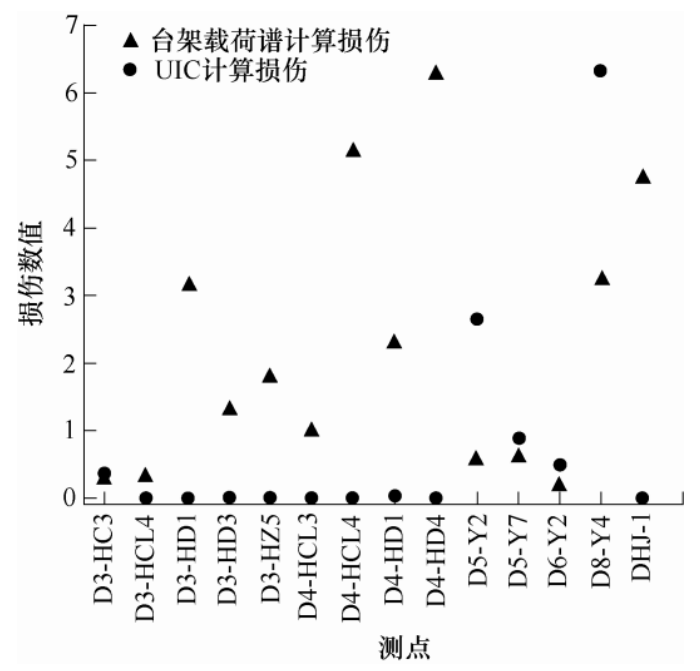

图 7 测点损伤对比
为进一步说明零部件载荷的影响, 在采用 UIC 标准加载主要运营载荷的基础上继续加载局部运营 载荷, 得到的损伤如表 11 所示。由表 11 可见, 加 上局部运营载荷之后, 齿轮箱吊座、电机吊座、制 动吊座以及减振器吊座处测点的损伤均有约 2 个数 量级的提升, 其余部位影响不大, 进一步验证了本 文的假设。然而对比实测载荷谱计算损伤可知, 这 些部位总损伤仍远小于实测损伤。以上结果表明, UIC 标准具有较好的普适性和精确性, 但由于研究 人员在制定标准时，以国外线路条件为基础，因而 未明确符合我国实际线路工况和运用特征所决定的 构架真实载荷输入, 所以出现了在某些线路上出现 地铁构架疲劳失效的现象; 基于实测数据的多轴加 载台架谱编制方法在构架损伤的评估精度方面接近 线路实测结果，在线路条件变化不大的前提下，可 以针对结构相似的构架进行疲劳评估及结构改进。

表 11 UIC 主要载荷和局部载荷计算损伤

\begin{tabular}{cc}
\hline 测点编号 & UIC 计算损伤 \\
\hline D3-HC3 & $3.91 \times 10^{-1}$ \\
D3-HCL4 & $1.62 \times 10^{-1}$ \\
D3-HD1 & $4.50 \times 10^{-1}$ \\
D3-HD3 & $2.10 \times 10^{-1}$ \\
D3-HZ5 & $2.90 \times 10^{-1}$ \\
D4-HCL3 & $1.10 \times 10^{-1}$ \\
D4-HCL4 & $3.40 \times 10^{-1}$ \\
D4-HD1 & $3.20 \times 10^{-1}$ \\
D4-HD4 & $7.00 \times 10^{-2}$ \\
D5-Y2 & $2.78 \times 10^{+0}$ \\
D5-Y7 & $9.10 \times 10^{-1}$ \\
D6-Y2 & $5.20 \times 10^{-1}$ \\
D8-Y4 & $6.34 \times 10^{+0}$ \\
DHJ-1 & $2.40 \times 10^{-1}$ \\
\hline
\end{tabular}

\section{6 结论}

（1）基于多轴振动疲劳理论推导了频域内恒幅 载荷和应力响应的计算方法, 克服了传统时域内分 立载荷谱计算损伤丢失了载荷耦合作用的弊端。

（2）分析了相关性与载荷相位之间的关系，将载 荷间的实测随机相位转化成台架试验固定相位, 得到 了可以实现多轴加载的地铁构架恒幅载荷程序谱。

（3）基于损伤一致性原则校准后的载荷谱计算 损伤速率已覆盖实测损伤速率, 比值范围在 1.04 6.47 , 表明多通道加载的台架载荷谱可以达到线路 测试同等条件的考核标准。

（4）分别采用频域法和时域法计算测点损伤, 
计算误差带在 $8.33 \%$ ～18.60\%，表明基于频域法的 试验台多通道加载方案, 可以准确反映载荷耦合作 用下测点的应力响应。

\section{参 考 文 献}

[1] 卢耀辉, 向鹏霖, 曾京, 等. 高速列车转向架构架动应 力计算与疲劳全寿命预测 [J]. 交通运输工程学报, 2017, 17(1): 62-70.

LU Yaohui, XIANG Penglin, ZENG Jing, et al. Dynamic stress calculation and fatigue whole life prediction of bogie frame for higlrspeed train[J]. Journal of Traffic and Transportation Engineering, 2017, 17(1): 62-70.

[2] 邹骅. 城际动车组转向架构架载荷谱研究[D]. 北京: 北 京交通大学, 2016.

ZOU Hua. The study on intercity EMU bogie frame load spectrum[D]. Beijing: Beijing Jiaotong University, 2016.

[3] 李凡松, 邬平波, 曾京. 车下设备承载结构疲劳试验载 荷谱编制方法[J]. 机械工程学报, 2016, 52(24): 99-106. LI Fansong, WU Pingbo, ZENG Jing. Compilation method of fatigue test load spectrum for underfloor equipment bearing structure[J]. Journal of Mechanical Engineering, 2016, 52(24): 99-106.

[4] HAN J W, KIM H S, BANG J S, et al. Fatigue strength evaluation of bogie frame of urban maglev train[J]. Engineering Failure Analysis, 2013，31(7): 412-420.

[5] LU Yaohui, ZHENG Heyan, ZENG Jing, et al. Fatigue life reliability evaluation in a high-speed train bogie frame using accelerated life and numerical test[J]. Reliability Engineering and System Safety, 2019, 24(13): 221-232.

[6] 邹骅, 李强, 孙守光. 基于载荷标定的城际列车转向架 载荷及应力分布特征研究[J]. 铁道学报, 2016, 38(10): 27-33.

ZOU Hua, LI Qiang, SUN Shouguang. Study on intercity train load spectrum distribution sstimation and calibration methods based on load demarcation[J]. Journal of the China Railway Society, 38(10): 28-33.

[7] 朱宁. 高速列车转向架结构损伤一致性载荷谱理论研 究[D]. 北京: 北京交通大学, 2016 .

ZHU Ning. Theoretical research of damage consistency load spectra on bogie frame structures of high-speed trains[D]. Beijing: Beijing Jiaotong University, 2016.

[8] 陈道云, 孙守光, 李强. 高速列车载荷谱推断及扩展方 法研究[J]. 机械工程学报, 2018, 54(10): 151-155. CHEN Daoyun, SUN Shouguang, LI Qiang. Study on deduction and extend of high-speed train load spectrum[J]. Journal of Mechanical Engineering, 2018, 54(10): 151-155.

[9] 董博, 王萌. 外部载荷对轨道车辆转向架构架结构静强 度影响程度的评估方法[J]. 中国铁道科学, 2018 , 39(6): 98-103.

DONG Bo, WANG Meng. Evaluation method for influence degree of external load on static strength of railway vehicle bogie frame structure[J]. China Railway Science, 2018, 39(6): 98-103.

[10] BENASCIUTTI D, CRISTOFORI A, TOVO R. Analogies between spectral methods and multiaxial criteria in fatigue damage evaluation[J]. Probabilistic Engineering Mechanics, 2013, 31(31): 39-45.

[11] CARPINTERI A, FORTESE G, RONCHEI C, et al. Spectral fatigue life estimation for non-proportional multiaxial random loading[J]. Theoretical and Applied Fracture Mechanics， 2016，83(2): 67-72.

[12] PITOISET X, PREUMONT A. Spectral methods for multiaxial random fatigue analysis of metallic structures[J]. International Journal of Fatigue， 2000， 22(7): 541-550.

[13] BRACCESR C, CIANETTI F, TOMASSINI L. Random fatigue: A new frequency domain criterion for the damage evaluation of mechanical components[J]. International Journal of Fatigue, 2015， 70: 417-427.

[14] BENASCIUTTI D, TOVO R. Comparison of spectral methods for fatigue analysis of broad-band Gaussian random processes[J]. Probabilistic Engineering Mechanics, 2006, 21(4): 287-299.

[15] 刘沫, 冯咬齐, 何玲. 卫星产品多轴随机振动试验条件 制定方法初探[J]. 航天器环境工, 2013, 30(2): 155-159. LIU Mo, FENG Yaoqi, HE Ling. The determination of conditions of multi-axis random vibration tests for satellite products[J]. Spacecraft Environment Engineering, 2013, 30(2): 155-159.

[16] CRISTOFORI A, SUSMEL L, TOVO R. A stress invariant based criterion to estimate fatigue damage under multiaxial loading[J]. International Journal of Fatigue, 2008, 30(7): 1646-1658.

[17] 王斌杰, 孙守光, 李强, 等. 基于载荷谱提升转向架构 架疲劳可靠性研究[J]. 铁道学报, 2019, 41(2): 23-30. WANG Binjie, SUN Shouguang, LI Qiang, et al. Research on the improvement of speed increased passenger car bogie frame reliability based on load spec- 
trum[J]. Journal of the China Railway Society, 2019, 41(2): 23-30.

[18] MIRJALILI S, LEWIS A. Grey wolf optimization[J]. Advances in Engineering Software, 2014, 69(7): 46-61.

[19] 张立. 北京地铁 2 号线车辆转向架构架载荷测试与研 究[D]. 北京: 北京交通大学, 2017.

ZHANG Li. Load test and study on bogie frame of
Beijing subway line 2[D]. Beijing: Beijing Jiaotong University, 2017.

作者简介: 张子璠, 男, 1992 年出生, 博士研究生。主要研究方向为轨 道车辆结构强度可靠性及载荷谱。

E-mail: 14116349@bjtu.edu.cn

杨广雪(通信作者), 男, 1982 年出生, 博士, 副教授, 硕士研究生导师。 主要研究方向为动应力测试、数据处理和载荷谱。

E-mail: gxyang@bjtu.edu.cn 